\title{
3D Numerical Simulation of Aerodynamic Characteristics of a Gas Filter
}

\author{
Fangfang Si¹,2, Pengfei Lian³, Derui Yang², Guolin Han², Shaoyue Hao³, Pingwei Ye ${ }^{1,2}$ \\ ${ }^{1}$ State Key Laboratory of NBC Protection for Civilian, Beijing, China \\ ${ }^{2}$ Research Institute of Chemical Defense, Beijing, China \\ ${ }^{3}$ Shanxi Xinhua Chemical Co. Ltd., Taiyuan, China \\ Email: siff0507@163.com
}

How to cite this paper: Si, F.F., Lian, P.F., Yang, D.R., Han, G.L., Hao, S.Y. and Ye, P.W. (2019) 3D Numerical Simulation of Aerodynamic Characteristics of a Gas Filter. Journal of Applied Mathematics and Physics, 7, 1920-1928.

https://doi.org/10.4236/jamp.2019.78132

Received: July 13, 2019

Accepted: August 25, 2019

Published: August 28, 2019

\begin{abstract}
The filter paper and activated carbon which filled inside the gas filter have porous media characteristics. In order to study the flow field structure in the filter layer and the activated carbon layer, Computational Fluid Dynamics method is used to simulate the aerodynamic characteristics of a simplified gas filter. The inertial and viscosity parameters of porous media are solved by Forchheimer equation. The three-dimensional N-S equation and the modified low Reynolds number $k-\varepsilon$ turbulence model are adopted to analyze the influence of the explosion-proof plate on the gas filter's aerodynamic characteristics. The results showed that the air age in the upstream of the activated carbon plates was small, which easily caused the rapid penetration of the Poisonous gas. And in the downstream and around of the activated carbon plates, the air age is larger, formed dead zone, the utilization rate of activated carbon is lower. The explosion-proof plate increases the pressure drop of the gas filter, the air age distribution in the filter layer is more uniform and the utilization rate of filter paper is improved. However, the explosion-proof plate has little influence on the flow field structure of the activated carbon layer.
\end{abstract}

\section{Keywords}

Numerical Simulation, Gas Filter, Air Age, Porous Media

\section{Introduction}

The gas filter in the collective protection equipment is mainly used in the ventilation system of civil air defense engineering, which can effectively filter the poisonous smoke, poisonous fog, biological aerosol and radioactive dust in the poisoned air, and provide clean air to the works. There are two kinds of gas filter 
structures: radial flow type and direct flow type. The radial flow gas filter is cylindrical. The direct flow filter is square, and the internal activated carbon plate is arranged in a v-shape. The internal structure of direct flow gas filter is very complex, including shell, explosion proof plate, filter layer, activated carbon layer, etc.

Due to the large size of the gas filter, it is easy to cause low utilization rate of filter paper and activated carbon, and the toxic gas quickly penetrates through the gas filter, reducing the protection time and causing energy waste. In order to improve the utilization rate of filter paper and activated carbon, extend the protection time and achieve the goal of reducing cost and risk, the structure of a gas filter can be optimized. The aerodynamic characteristics of gas filter must be considered when optimizing its structure.

To study the aerodynamic characteristics of a gas filter, it is mainly to study the flow field structure inside the filter layer and the activated carbon layer, especially the airflow distribution inside the activated carbon layer. Filter paper and activated carbon are the main filter materials for filling the gas filter. Both filter paper and activated carbon have porous media characteristics. Experiment method cannot look inside the porous medium such as activated carbon and filter air distribution. With the rapid development of computer and the numerical methods, exploring the aerodynamic characteristics of a flow field using computational fluid dynamic (CFD) tools is helpful in analyzing the flow variables in porous media within a gas filter [1] [2] [3] [4]. The preferential flow (adsorption failure due to excessive adsorption) and dead zone (due to gas does not flow through or residence time is too long lead to insufficient adsorption or basic no adsorption) area. Yin Chia Su and Chun Chi Li et al. used CFD method to simulate a gas mask canister [5] [6] [7], and the research results were in good agreement with the measured results, providing theoretical basis for the structure optimization of gas mask canister. However, very little existing literature analyzes the aerodynamic characteristics of a gas filter. Therefore, the topic of the aerodynamic behavior of a gas filter with two kinds porous materials is worthy of further research.

The research and development of gas filter in our country basically follows the previous structural design, which is mainly based on experience and actual measurement, and lacks the research on aerodynamic characteristics, which makes the researchers have only a partial understanding of the experimental results and difficult to analyze in depth theoretically, which seriously restricts the optimization of gas filter. Therefore, the computational fluid dynamics method was used to study the aerodynamic characteristics of the simplified rfp-1000gas filter with or without explosion-proof plate. The numerical simulation results can provide theoretical basis for the optimization of gas filter structure.

\section{Problem}

\subsection{Governing Equations}

The governing equation is a 3D Reynolds mean Navier-Stokes equation, which 
can be written as follows for 3D flows.

$$
\frac{\partial}{\partial x_{i}}\left(\rho u_{i} u_{j}\right)=-\frac{\partial p}{\partial x_{i}}+\frac{\partial \tau_{i j}}{\partial x_{j}}+\rho g_{i}-\frac{\partial}{\partial x_{j}}\left(\rho \overline{u_{i}^{\prime} h_{j}^{\prime}}\right)+S_{i}
$$

where $\rho$ is the gas density; $u_{i}$ is the velocity component in direction of $i ; p$ is the pressure; $\tau_{i j}$ is the viscosity shear stress tensor; $g_{i}$ is the acceleration due to gravity in $i$ direction. When flow is not laminar flow, Reynolds stress term $\rho \overline{u_{i}^{\prime} h_{j}^{\prime}}$ is associated with the mean flow of Boussinesq hypothesis. The source term $S_{i}$ describes the pressure gradient in the porous medium, which is assumed to be isotropic.

The flow rate of the rfp-1000 gas filter is $1000 \mathrm{~m}^{3} / \mathrm{h}$, with a Reynolds number of 78,000. Therefore, it is necessary to consider the influence of turbulence and adopts a modified low Reynolds number $k$ - $\varepsilon$ turbulent model. The working gas is air, for which $\rho=1.225 \mathrm{~kg} \mathrm{~m}{ }^{-3}, \mu=1.7894 \times 10^{-5} \mathrm{~kg} \cdot \mathrm{m}^{-1} \cdot \mathrm{s}^{-1}$.

In order to study the preferential flow and dead zone in the porous media region, and solving the transient adsorption mode requires a long computation time. Therefore, air age index is used to investigate aerodynamic behavior within the gas filter. The definition of air age is the local mean age of the fluid, or local mean residence time, which is the average time that a fluid particle takes to reach any point of the domain from the gas filter inlet. A greater air age represents a longer residence time and thus a greater chance for adsorption. However, a great air age means that the air has difficulty flowing through the space, thus forms a dead zone. Using the flow variable distributions described by the air age makes it much easier to determine the position of dead zones by observing the flow structure within porous media [5]. The air age equation is:

$$
\frac{\partial}{\partial x_{i}}\left(\rho u_{i} \tau\right)=\frac{\partial}{\partial x_{i}}\left(\frac{\mu_{\mathrm{eff}}}{\sigma_{\tau}} \frac{\partial \tau}{\partial x_{i}}\right)+\rho
$$

where $\tau$ is the air age; $\mu_{\text {eff }} / \sigma_{\tau}$ is the local actual diffusivity in turbulent model; $\mu_{\text {eff }}$ $=\mu_{l}+\mu_{t}$ ( $\mu_{\text {eff }}$ is effective viscosity, $\mu_{l}$ is molecular and $\mu_{t}$ is turbulent viscosity); $\sigma_{\tau}$ $=1$ is the turbulent Schmidt number.

\subsection{Boundary Conditions}

The volumetric flow rate of gas filter inlet is constant in all simulations, $\mathrm{Q}=$ $1000 \mathrm{~m}^{3} / \mathrm{h}$. At the inlet, the reference pressure is $101,325 \mathrm{~Pa}$. The outlet boundary condition is the pressure outlet boundary. The no slip boundary is assumed for all solid walls.

\subsection{Numerical Method}

The study uses the FLUENT flow solver. The convection term is discredited with the second-order upwind scheme and the viscosity term is discredited with a second-order central differential scheme. The COUPLE algorithm is the solution algorithm for pressure-velocity coupling. 


\subsection{Grid and Model}

Two simplified structural forms of gas filter with or without explosion-proof plate are studied. The model without explosion-proof plate is defined as model A, and the model with explosion-proof plate is defined as model B, as Figure 1 and Figure 2 shows.

To simplify the computation, this study omits the effect of multi-pleated filter paper and treats the filter layer as a single layer. The two porous layers are assumed to be homogeneous and isotropic. For computational efficiency, only $1 / 2$ of the full $3 \mathrm{D}$ model is simulated. Unstructured tetrahedral mesh is used. Figure 3 displays the grid of model A.

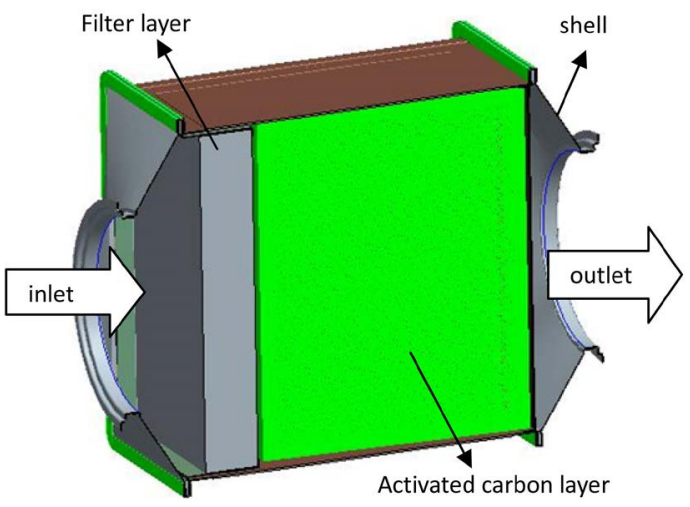

Figure 1. The gas filter without explosion-proof plate (model A).

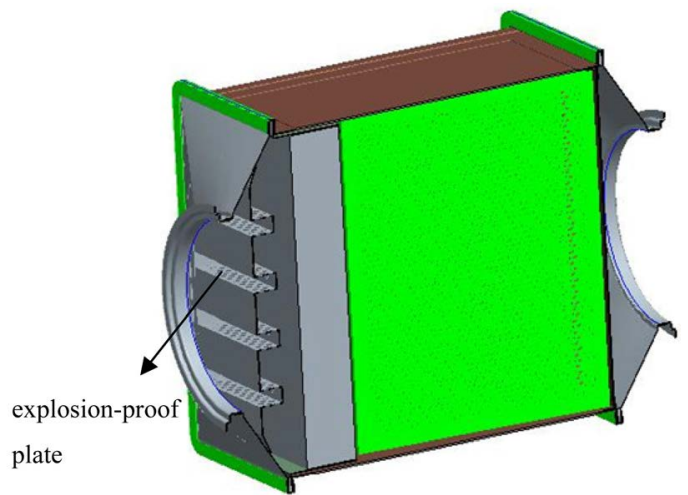

Figure 2. The gas filter with explosion-proof plate (model B).

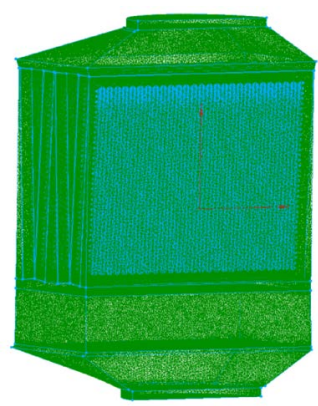

Figure 3. The grid of model A. 


\subsection{Porous Media Parameters}

The pressure drop of the gas filter mainly comes from filter paper and activated carbon. Since the Reynolds number of rfp-1000 gas filter is 78,000, not only the influence of viscosity but also the inertial effect should be considered, so the Forchheimer equation is used to describe the momentum dissipation of fluid flowing through porous media:

$$
-\frac{\Delta p}{L}=\alpha \mu V_{s}+\beta \rho V_{s}^{2}
$$

where $\alpha$ is the reciprocal permeability of the porous material, or viscosity parameter. $\beta$ is usually called the inertial parameter. $\Delta P$ is the pressure drop of the porous medium zone. $L$ is the length of the flow direction; $\mu$ is the fluid viscosity. $V s$ denotes the superficial velocity of the fluid entering the porous medium zone.

The filter paper and activated carbon used in the rfp-1000 gas filter are consistent with the references [8]. Therefore, the coefficients of the filter layer and the activated carbon layer are consistent with the references [8].

The coefficients for the filter layer are determined as

$$
\alpha_{1}=2.19 \times 10^{9} \mathrm{~m}^{-2}, \beta_{1}=7.5 \times 10^{4} \mathrm{~m}^{-1}
$$

The coefficients for the activated carbon layer are determined as

$$
\alpha_{2}=2.03 \times 10^{9} \mathrm{~m}^{-2}, \beta_{2}=7.5 \times 10^{4} \mathrm{~m}^{-1}
$$

\section{Results and Discussion}

\subsection{Empty Gas Filter Simulation}

The aerodynamic characteristics of the gas filter without filling filter paper and activated carbon are numerically simulated.

Figure 4 shows the spatial streamlines colored by velocity of model A and model B. The results reveal that when the model hasn't explosion-proof plate, large backflow vortexes are formed in the filter layer and the airflow velocity

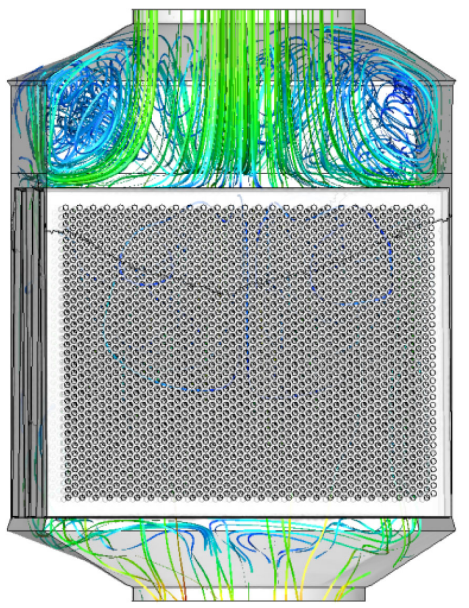

(a)

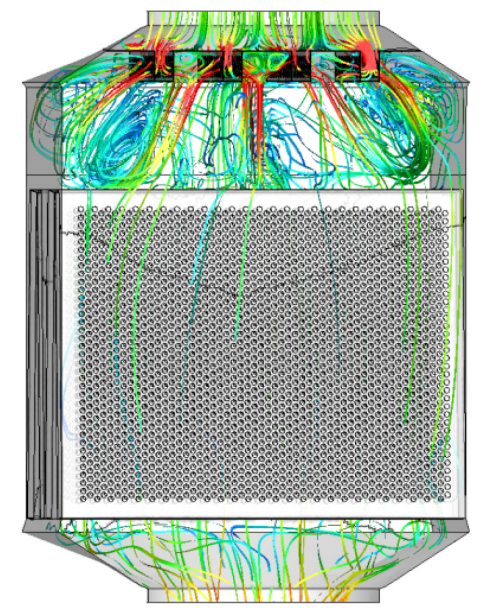

(b)

Figure 4. A spatial stream line colored by velocity. (a) Model A; (b) Model B. 
decreases. When the fluid flows actually through the porous medium, the porous flow will not cause backflow in the peripheral zone. However, if the fluid is not easy to flow into the zone or has a low velocity in the zone, a dead zone forms. In turn, this reduces the flow-through area. When the model has an explosion-proof plate, due to the extreme change of geometric structure, the flow suddenly expands and the airflow velocity increases. After touching the shell wall, a backflow vortex is formed in the filter layer. The backflow zone of model B is smaller than model A.

\subsection{Air Age}

The aerodynamic characteristics of model A and B with filling filter paper and activated carbon are simulated.

The influence of explosion-proof plate on the pressure drop and flow field structure of the gas filter is studied. The pressure drop of model A is $605 \mathrm{~Pa}$, and that of model B is $617 \mathrm{~Pa}$. The explosion-proof plate increases the pressure drop of the gas filter. The experimental result of the gas filter with explosion-proof plate is $640 \mathrm{~Pa}$. The simulated pressure drop is close to the experimental value.

Figure 5 shows the air age contours of model A and B. The air age around the activated carbon plates is relatively large, indicating that the airflow through around the activated carbon plates is needed for a long time, thus forming a dead zone. There is a big difference between the air age of model A and model B in the filter layer. Model A has a smaller air age in the central area of the filter layer, and the airflow passes through the central area quickly. The air age in the square corner area of model A is relatively larger, forming a dead zone. The air age in the square corner area in the filter layer of model B is also larger, but the dead zone range of model A is smaller than that of model A. The difference of air age between the center area and around area is smaller, and the utilization rate of filter paper is improved.

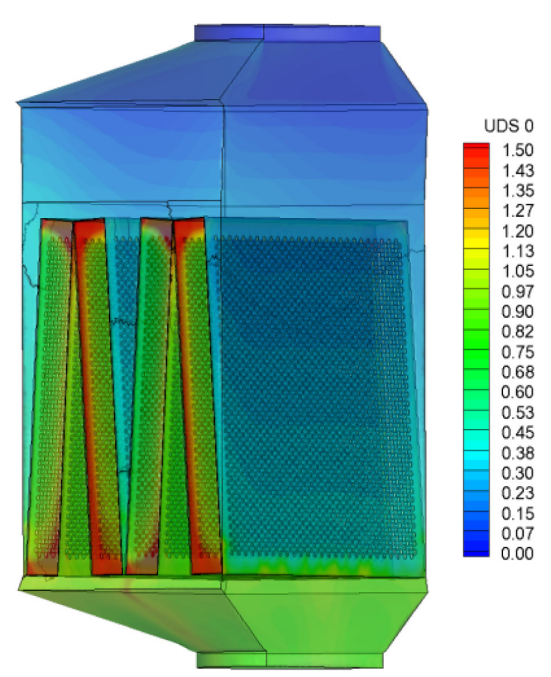

(a)

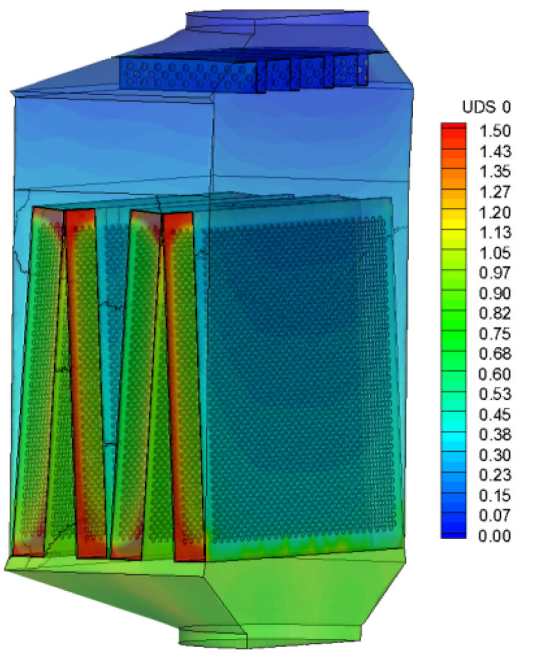

(b)

Figure 5. Air age contours for the gas filter. (a) Model A; (b) Model B. 
Figure 6 and Figure 7 show the air age contour on $\mathrm{Z}$-axis and X-axis sections of the activated carbon plate. Along the flow direction ( $Z$ direction), the air age value in the upper $1 / 3$ area of the activated carbon plates is smaller, and the air flow rapidly passes through the upstream area of the activated carbon plates, which is easy to cause rapid penetration of poison. However, along downstream area of the activated carbon plates, the air age gradually increases. The residence time of air in this area is longer, forming dead zone. The utilization of activated carbon is insufficient. Along the $\mathrm{x}$ direction, the air age in the central area of the activated carbon plates is smaller, while the air age in the two sides gradually increases. The activated carbon plates form dead zone near the shell, and the utilization rate of activated carbon decreases. By comparing the air age distribution of model $\mathrm{A}$ and model $\mathrm{B}$, it can be seen that the explosion-proof plate has little influence on the structure of the activated carbon layer flow field.

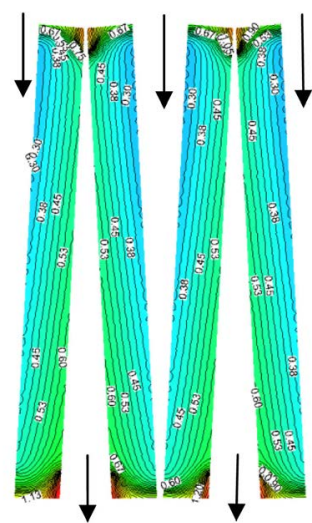

(a)

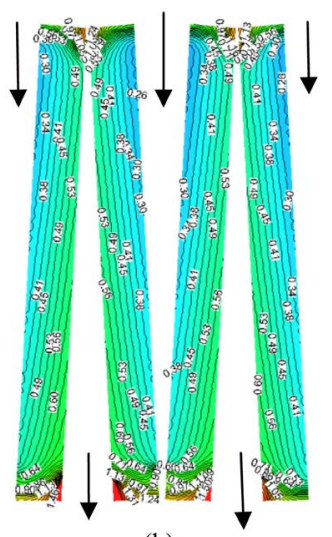

(b)

Figure 6. Air age contours on $x=0$ section of the activated carbon layer. (a) Model A; (b) Model B.

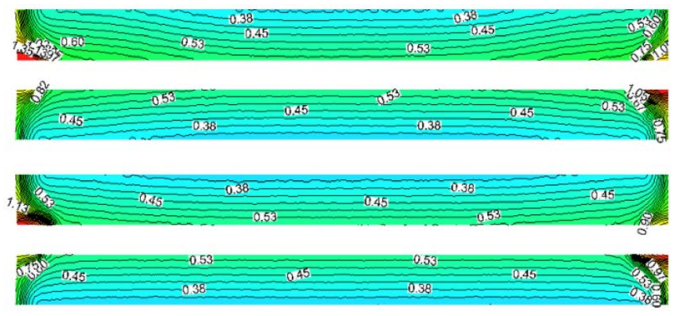

(a)

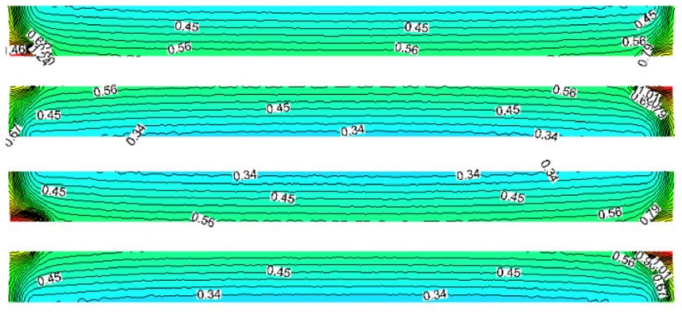

(b)

Figure 7. Air age contours on $z=0$ section of the activated carbon layer. (a) Model A; (b) Model B. 


\section{Conclusions}

In this paper, CFD method is used to study a simplified rfp-1000 gas filter with a very complex inner configuration of two porous media. The influence of explosion-proof plate on the aerodynamic characteristics of gas filter was investigated.

The numerical simulation results reveal that, when the gas filter without porous media and explosion-proof plate, a large backflow vortex is formed in the filter layer, and the airflow velocity decreases. When the gas filter has explosion-proof plate, the airflow speed increases, a backflow vortex is formed also, but the backflow vortexes are smaller than those without explosion-proof plate. Filling with filter paper and activated carbon, the air age value in the upper $1 / 3$ area of the activated carbon plates is smaller, which is easy to cause rapid penetration of the poison. The downstream and around of the activated carbon plates, the air age value is larger, formed dead zone. Activated carbon is not fully used. The air age distribution in the filter layer of gas filter with explosion-proof plate is more uniform and the utilization rate of filter paper is improved. However, the explosion-proof plate has little influence on the flow field structure of the activated carbon layer. The results of this study will be helpful in designing gas filter, and reveals that the development of a gas filer with high utilization rate of the porous media is feasible. The influence of other components on the aerodynamic characteristics of the gas filter will be further optimized.

\section{Conflicts of Interest}

The authors declare no conflicts of interest regarding the publication of this paper.

\section{References}

[1] Subrenat, A., Bellettre, J. and Le Cloirec, P. (2003) 3-D Numerical Simulations of Flow in a Cylindrical Pleated Filter Packed With Activated Carbon Cloth. Chemical Engineering Science, 58, 4965-4973. https://doi.org/10.1016/j.ces.2003.07.012

[2] Baléo, J.N. and Le Cloirec, P. (2000) Numerical Simulation of the Spatial Distribution of Mean Residence Time in Complex Flow through Porous Media. Progress of Theoretical Physics, 138, 690-695. https://doi.org/10.1143/PTPS.138.690

[3] Baléo, J.N., Subrenat, A. and Le Cloirec, P. (2000) Numerical Simulation of Flows in Air Treatment Devices Using Activated Carbon Cloths Filters. Chemical Engineering Science, 55, 1807-1816. https://doi.org/10.1016/S0009-2509(99)00441-8

[4] Mößner, M. and Radespiel, R. (2017) Flow Simulations over Porous Media-Comparisons with Experiments. Computers and Fluids, 1-13. https://doi.org/10.1016/j.compfluid.2017.03.002

[5] Li, C.C. and Su, Y.C. (2009) CFD Simulation of a Gas Mask Filter in Three Realistic Breathing Patterns. Journal of Flow Visualization \& Image Processing, 16, 201-219. https://doi.org/10.1615/JFlowVisImageProc.v16.i3.10

[6] Li, C.C. (2009) Aerodynamic Behavior of a Gas Mask Canister Containing Two Porous Media. Chemical Engineering Science, 64, 1832-1834. https://doi.org/10.1016/j.ces.2009.01.009 
[7] Su, Y.C. and Li, C.C. (2015) Computational Fluid Dynamics Simulations and Tests for Improving Industrial-Grade Gas Mask Canisters. Advances in Mechanical Engineering, 7, 1-14. https://doi.org/10.1177/1687814015596297

[8] Si, F.F., Ye, P.W., Huang, F., Xi, L., Wang, L.Y. and Yang, D.R. (2019) 3D Numerical Simulation of the Aerodynamic Characteristics of Arc Canister. Journal of Ordnance Equipment Engineering, 40, 28-33. 